\title{
Modeling Rain Attenuation Effect in Free Space Optic Propagation
}

\author{
A K Rahman \\ Department of Electrical and Electronics \\ Universiti Malaysia Sarawak \\ Kuching, Malaysia \\ karahman@unimas.my
}

\author{
Tauffiq Khirham \\ Department of Electrical and Electronics \\ Universiti Malaysia Sarawak \\ Kuching, Malaysia \\ aapenqq@gmail.com
}

\begin{abstract}
The project is based on analyzing various intensity of rain through the transmission in free space optic (FSO) communication. The idea is to verify how characteristics of rain can affect the FSO communication system. The required software for simulation of this project is Optisys software system. The locations chosen for this project are Kuching and Samarahan. The rain data is officially collected from Malaysia Meteorological Department (MMD). The measurement through simulation using the real parameter from Laserbit system. The result will determine how intensity of rain can impair the link and to find the alternative method to overcome this problem.
\end{abstract}

Keywords—intensity, transmission, simulation, impair

\section{INTRODUCTION}

Optical wireless communications are based on the data transmission through the atmosphere using optical carriers. Optical links can be very short in range (in the order of $\mathrm{cm}$ ), as well as long range (hundreds of $\mathrm{km}$ ). Hence, the terms freespace optic or well known as FSO typically refer to outdoor terrestrial links operating over distances from a few tens of meters up to several kilometers. The use of FSO is highly recommended in the telecommunication community for applications that demand capability of very large bandwidth.

The advantages of FSO communication system over conventional fiber and microwave links are low costs of equipment, flexible and license-free installation [1]. The propagation of laser beams in the atmosphere is highly affected by aerosol scattering and atmospheric turbulence which limit the link availability on the path length. These effects are much worse than microwave relay links issue. In details, impairments are due to (i): air turbulence that caused beam spreading, beam wander, scintillation and degradation of the coherence of wave front [2], and (ii): hydrometeors (rain and snow) and suspended water particles (fog is the worst case for FSO system) that contribute to more losses due to scattering [3]. The type of scattering is determined by the size of the particles. To analyze the scattering effect, it is more suitable to utilize the geometric optics.

The FSO communication link performance is measured through its availability. The tropical climate rainfall has become the major factor that affects the link availability of FSO. The link availability is estimated due to the rainfall impact. The attenuation due to rainfall will cause the scattering effect. The estimation is based on the rain attenuation model which correspond to the rainfall rate $(\mathrm{mm} / \mathrm{hr})$. The target is to predict the maximum rainfall rate against the link ranges. Previous researchers have been investigating to determine the rainfall attenuation level and relating it to the rain rate [4], [5], [6], [7]. However, the scope of work focused more on European weather, and minimal effort has been channeled to investigate the work in the tropical region, which experiences rainfall at very high rate. An almost-daily rainfall which contain between 1500 and $2000 \mathrm{~mm}$ annually, was certain to encourage the quality transmission of FSO link deployed. This effort will address the vacancy to a significant extent, with precise mathematical relations, derived and improved over the years by various researchers [8] coupled by simulations under the OptiSystem software.

\section{THEORETICAL APPROACHES}

\section{A. Scattering Coefficient}

Rain is precipitation droplets that condensed from atmospheric water vapour. When the droplets of rain become heavy, it will fall under gravity. Rain is under geometrical scattering where it occurs in the lower portion of the atmosphere when the particles are larger than the incident radiation. The scattering coefficient can be calculated by using Stroke Law [3].

$$
\beta_{\text {rainscat }}=\pi a^{2} N_{a} Q_{\text {scat }}\left(\frac{a}{\lambda}\right)
$$

Where $a$ = radius of raindrop $(0.001 \mathrm{~cm}$ to $0.1 \mathrm{~cm}), N_{a}=$ rain drop distribution, $Q_{\text {scat }}=$ scattering efficiency, $\lambda=$ wavelength

\section{B. Atmospheric Attenuation}

The attenuation of laser power in the atmosphere is described by Beer's Law [9].

$$
\tau(R)=-\frac{P(R)}{P(O)}=e^{-(\beta) R}
$$

